\title{
Dispersion of the nonlinear susceptibility in gold nanoantennas
}

\author{
V. Knittel, M. P. Fischer, M. Vennekel, T. Rybka, A. Leitenstorfer, and D. Brida* \\ Department of Physics and Center for Applied Photonics, University of Konstanz, D-78457 Konstanz, Germany
}

\begin{abstract}
Femtosecond optical pulses tunable in the near infrared are exploited to drive third harmonic generation (THG) and incoherent multiphoton photoluminescence (MPPL) in gold plasmonic nanoantennas. By comparing the yield of the two processes concurrently occurring on the same nanostructure, we extract the coherent third-order response of the antenna. Its contribution is enhanced at shorter excitation wavelengths allowing the observation of dispersion in the nonlinear susceptibility of gold.
\end{abstract}

Plasmonic antennas are metallic nanostructures that allow the collection of light focused at the diffraction limit to further concentrate it at the nanometer scale [1,2]. While this process is affected by losses, the appeal of nanoantennas lies in their capability to enhance light-matter interactions at dimensions that are typical for elementary emitters [3-5] and in the fact that the augmented electromagnetic fields [6-10] can be harnessed to drive the nonlinear emission of new optical frequencies as a source of excitation directly in the near field. In particular, the study of the interplay between all the conversion mechanisms that lead to the generation of new photon energies in plasmonic nanostructures can unveil the effective nonlinear optical response of metals.

In a nanoantenna illuminated by a laser pulse, phenomena like harmonics generation [11-13], frequency mixing [14,15], and white-light continuum formation [16] are intrinsically coherent with respect to the driving light pulses since they originate from the nonresonant response of the electrons [4]. In contrast, multiphoton photoluminescence (MPPL) produced by the metallic nanostructures exposed to infrared illumination is incoherent and noninstantaneous since it follows the sequential absorption of single photons [17-19]. A quantitative assessment of the nonlinear response of plasmonic nanostructures and its underlying origin is hampered by several difficulties [20]. Firstly, the susceptibility of bulk metals, gold in particular, is complex to evaluate and several contradictory results are reported in the literature [21]. One reason for this discrepancy of results lies in the experimental techniques employed for the characterization. Methods like third-harmonic generation (THG) [22], Z-scan [23,24] and four-wave mixing [25] are, in fact, sensitive to different contributions (coherent and incoherent) of the susceptibility $[21,26,27]$. Secondly, the very same field enhancement that intensifies the nonlinear response at the nanoscale is heavily dependent on the geometrical properties of the plasmonic structures and it is extremely difficult to evaluate with precision, especially from the experimental point of view [6-10,20,28]. Definitely, the magnitude of the plasmonic enhancement must be known with high confidence for a precise analysis of the optical response since the efficiency of harmonics generation as well as of other multiphoton processes scales nonlinearly $[6,29]$.

*Corresponding author: daniele.brida@ uni-konstanz.de
Here, we circumvent this problem by comparing the yields of third-harmonic generation (coherent) [2,30] and multiphoton photoluminescence (incoherent and retarded) [31,32] in individual gold nanoantennas illuminated by femtosecond near-infrared pulses. This approach allows us to directly evaluate the susceptibility of gold since the field enhancement is identical for the two nonlinear processes that are measured experimentally on the very same emitter. Our results show a strong increase of the $\chi^{(3)}$ response when the driving optical field is tuned to higher frequencies, thus unveiling the dispersion in the coherent third-order nonlinearity of gold.

Experimentally, we focus on two key elements: the evaluation of the nonlinearity and the efficiency of the MPPL and THG process for different excitation energies. In addition, we spectrally resolve the nonlinear order of MPPL in contrast to former studies [6,7,16-20,28,29,31-34] where its value is treated as one single integer assigned to the spectrally integrated MPPL. This result highlights the complex chain of linear absorptions that brings the electrons out of thermal equilibrium thus leading to the MPPL emission [17,18,19].

As a driving source for the nonlinear emission in gold nanoantennas, we employ an ultrabroadband femtosecond fiber laser that guarantees the stability and broad spectral coverage necessary to study the dependence on the excitation wavelength and thereby measuring the dispersion of the nonlinear susceptibility [35-37]. The ultrashort pulses are generated in a highly nonlinear fiber that allows tuning of the center wavelength from 900 to $2100 \mathrm{~nm}$ at a repetition rate of $40 \mathrm{MHz}$. They are focused on a single resonant nanostructure with polarization set parallel to the long antenna axis by an all-reflective objective (Cassegrain geometry, NA 0.65). The nonlinear emission is then collected in transmission with a dark field objective (NA 0.95) and spectrally resolved $(150 \mathrm{~g} / \mathrm{mm}$ grating) on a CCD array $(1024 \times 256$ pixels $)$. The average power is set to $2.5 \mathrm{~mW}$ while the pulse bandwidth is limited to $10 \mathrm{THz}$. This optimization guarantees that, irrespective of the excitation frequency, the pulses are always compressed to the constant duration of $130 \mathrm{fs}$. Owing to the near-infrared excitation driven by the Er:fiber laser system [35-37], we are able to stimulate processes of high nonlinear orders (up to 6) with emission in the visible regime.

The object of this study are monocrystalline and polycrystalline gold nanorods fabricated via focused ion beam milling into single-crystal gold flakes [38] and via electron beam lithography with subsequent thermal evaporation of a gold film, respectively [see Fig. 1(a)]. These nanoantennas 
(a)

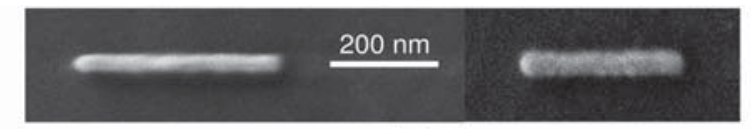

(b)
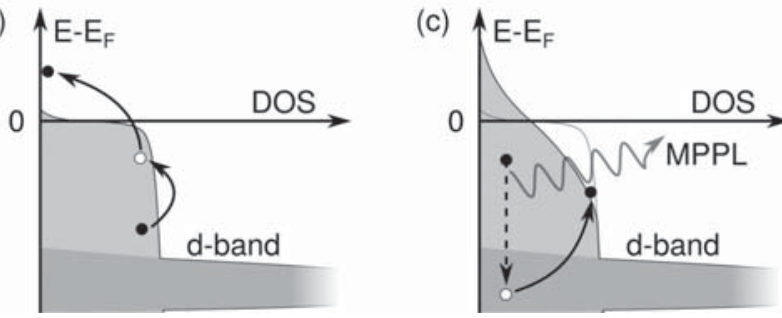

FIG. 1. (a) Scanning electron micrograph of two polycrystalline nanorods resonant at $2000 \mathrm{~nm}$ (left) and $1400 \mathrm{~nm}$ (right), used in the experiments. (b) Sketch of the density of occupied states in gold. The solid arrows depict the sequence of individual absorption steps that lead to a hot electronic distribution in the case of excitation with near-IR photons. (c) One additional photon promotes an electron from the $d$ band to states that become available below the Fermi level. The residual hole (white circle) then recombines (dashed arrow) with the consequent emission of PL that results from a multiphoton process.

have a thickness of $30 \mathrm{~nm}$, while a 2-nm chromium layer is used to facilitate adhesion onto the fused silica substrate (thickness $150 \mu \mathrm{m}$ ). The rod length is individually customtailored to tune its resonance in accordance to the excitation wavelength set by the laser pulses, while the width was fabricated to be $50 \mathrm{~nm}$. The simple rod geometry is chosen since it minimizes influences from the shape on the nonlinear emission spectrum [35] and is not subject to gap variations that strongly affect the emission $[6,9,10]$. By investigating both mono- and polycrystalline gold nanostructures, we prove that crystallinity does not affect the fundamental mechanisms driving the nonlinear emission, in contrast to the spectral shape of the nonlinear emission [35].

THG from nanostructures is a prototypical third-order process, enabled by the contribution of $\chi^{(3)}$ in the emitting medium. It is a coherent phenomenon that is a direct consequence of the nonlinear polarization established in the antenna and thus it does not deposit energy into the system. In contrast, the incoherent PL is emitted via the radiative recombination of $s p$ electrons with holes formed in the $d$ band, as sketched in Figs. 1(b) and 1(c). Hence the incoming photons must directly excite $d$ electrons into the conduction band above the Fermi level. In the case of MPPL, the energy of a single near-IR photon does not allow for this excitation mechanism until the electronic bath is brought so strongly out of equilibrium that a significant density of unoccupied states occurs well below the Fermi energy. The prerequisite for this condition is a cascaded absorption of several photons that explains the nonlinear origin of subsequent PL emission [17-19], see Fig. 1(b). The sequence of intraband absorptions is possible since the requirement for momentum conservation is relaxed in nanoparticles where the dipole approximation is less stringent and a plasmonic excitation is present $[32,33]$. In our experiments, the wavelength of the driving pulses is tuned between 1200 and $2000 \mathrm{~nm}$ (i.e., photon energies from 0.6 to $1 \mathrm{eV})$, thus requiring a multiphoton process for the generation of PL.
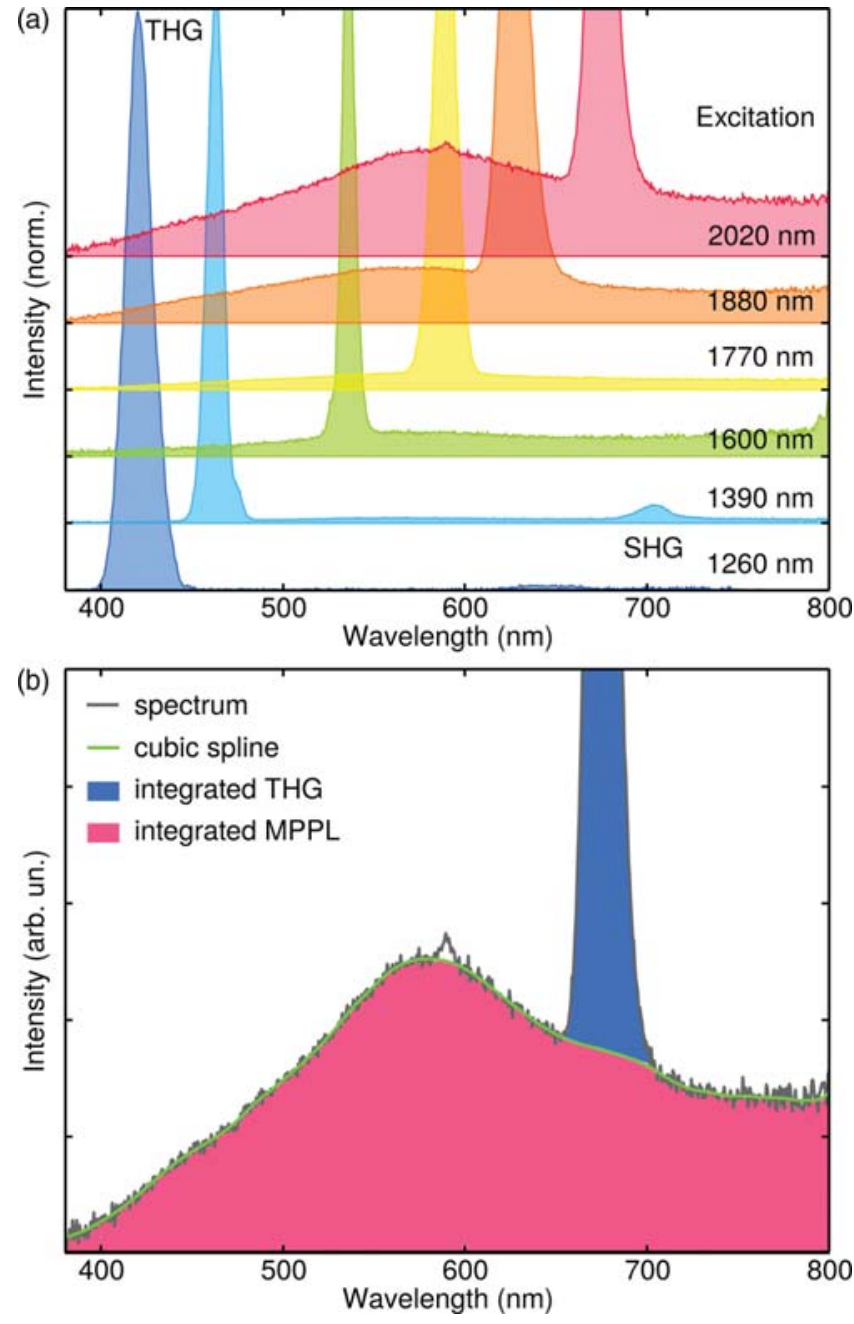

FIG. 2. (a) Typical nonlinear emission spectra obtained from single monocrystalline nanorods, each custom-tailored to be resonant to the excitation wavelength between 1260 and $2020 \mathrm{~nm}$. Consequently, the rod length varies from 170 to $360 \mathrm{~nm}$. The spectra are normalized to their narrow THG maximum, residing on the broad background of MPPL emission. Spurious second harmonic generation (SHG) may be observed if within the recorded spectral window. (b) Separation of the coherent THG emission (blue) from the incoherent MPPL (red) background in the nonlinear response (gray) of a monocrystalline nanorod with a cubic spline (green).

Typical nonlinear emission spectra obtained by resonantly exciting single monocrystalline nanorods at different wavelengths are displayed in Fig. 2. The optical pulses are tuned from 1200 to $2050 \mathrm{~nm}$, resulting in visible THG emission. All spectra are normalized to their dominant and narrow THG peak. Short excitation wavelengths can lead to weak SHG residual signals due to minor symmetry breaks in the geometry of the antennas and at surfaces [7,34]. In every case, broad MPPL is emitted but it is evident that its energy content relative to the THG intensity diminishes drastically while the excitation pulses are tuned towards the visible. The nonlinear emission from polycrystalline rods displays the same features and analogous dependence on the driving pulse wavelength. The measured spectra can be exploited for the separation of the 

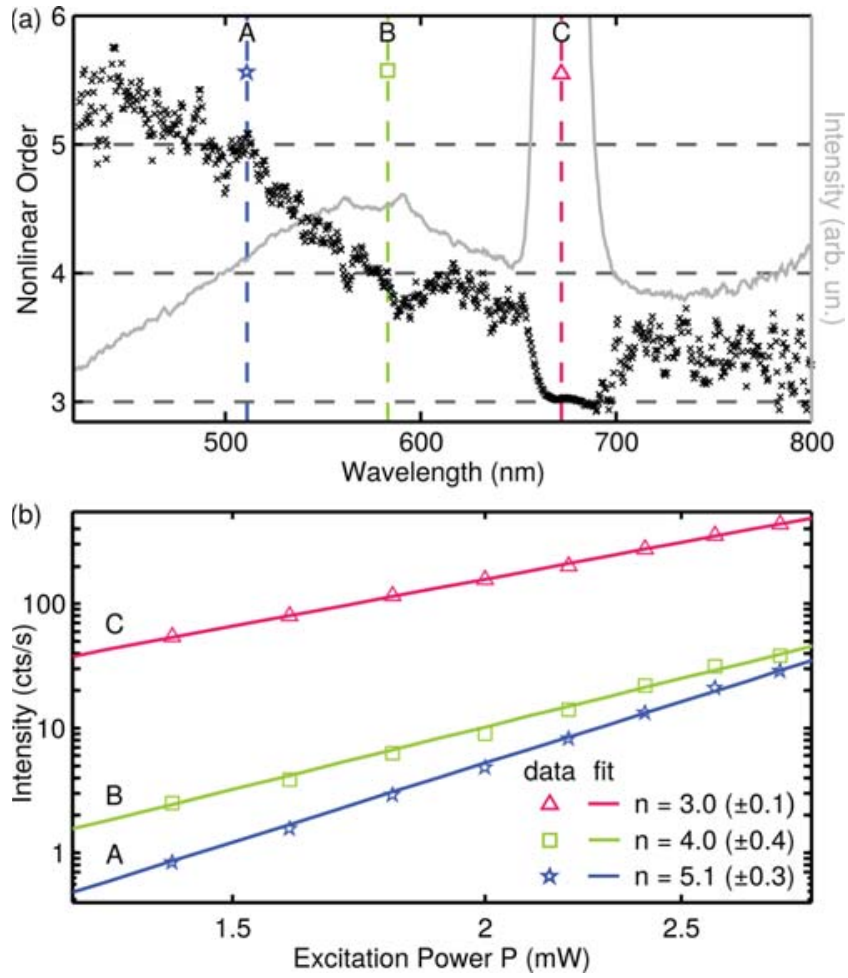

FIG. 3. Spectrally resolved power dependence of the nonlinear emission obtained from a gold nanorod of $320 \mathrm{~nm}$ length. (a) The nonlinear order $n$ of each corresponding emission wavelength in the case of a monocrystalline rod (black crosses) together with the nonlinear emission spectrum acquired at the maximum excitation power (gray). The excitation was tuned to a central wavelength of $2020 \mathrm{~nm}$. The vertical lines (A, B, and C) mark positions for which the power dependence fits are shown in (b): the experimental data points of emission intensity are depicted versus excitation power (symbols). The solid lines in this double-logarithmic graph show the least-square exponential fits determining the nonlinear order $n$ plotted in (a).

two contributions to the nonlinear emission. Figure 2(b) shows how a cubic spline (green) is fitted to the incoherent MPPL background (red) in order to extract the coherent THG peak (blue). Both nonlinear contributions can be integrated independently and then compared, as demonstrated in the following.

Figures 2 and 3(a) shows that the MPPL is observed down to a wavelength of $400 \mathrm{~nm}$. Therefore we expect nonlinear orders of at least $n=3$ or higher for excitation with wavelengths from 1200 to $2050 \mathrm{~nm}$. However, this simplified picture assumes complete conservation of radiation energy and neglects any dissipative coupling to the electronic system. In contrast, proper analysis should also account for the fact that the electrons might lose energy between two absorption events, thus effectively increasing the nonlinear order of the process. Even more importantly, the probability of creating a hole in the $d$ band is related to the global (nonequilibrium) distribution of the electrons in the whole conduction band. Consequently, it is not possible to define an exact sequence of a finite number of coherent absorption processes leading to the emission of a single PL photon. Instead, a fractional nonlinear order for MPPL may be observed (as in Refs. [28,29,31,34]), which must be understood as an effective number of photons averaged over several excitation pathways. To determine this nonlinear order, we performed a spectrally resolved analysis of the MPPL emission as a function of the excitation density. So far, solely the nonlinear order of the integrated PL spectrum was investigated [6,16-20,28,29,31-34] or restricted wavelength regions were compared [7].

To overcome this limitation, we measured a series of emission spectra $I_{\mathrm{em}}\left(\lambda, P_{\mathrm{exc}}\right)$ from a single antenna for increasing excitation power $P_{\text {exc }}$ and then fit a power law $I_{\mathrm{em}}\left(\lambda, P_{\mathrm{exc}}\right)=a(\lambda) P_{\mathrm{exc}}^{n(\lambda)}$ to the increasing intensity at each emission wavelength. $a(\lambda)$ and $n(\lambda)$ are the varied parameters with the latter corresponding to the nonlinear order. Its value is shown in Fig. 3(a) for a typical dataset obtained at an excitation wavelength of $2020 \mathrm{~nm}$ from a monocrystalline rod (black crosses). By observing the nonlinear order at the wavelengths that correspond to the THG emission we can extract a value of exactly 3 as expected from a fully coherent process. The power dependence is depicted in Fig. 3(b) (triangles and fit as solid red line).

At wavelengths corresponding to MPPL emission, the effective nonlinear order is found to be between 3 and 6 . Furthermore, we observe that the nonlinearity increases with the energy of the emitted photons. The increase appears rather smooth in the spectrum. This observation is compatible with the fact that radiative recombination is related to the probability of creating a hole in the $d$ band only for electrons below the Fermi level. Instead, above $2.4 \mathrm{eV}$ the joint density of states changes significantly with the temperature of the electronic system. Consequently, high-energy photon PL stems from the exponential tail of the Fermi-Dirac distribution that describes the hot electron bath and thus displays a higher effective nonlinear order. These observations are valid also for polycrystalline rods proving once more that, while the PL spectrum can be shaped by the geometry of the plasmonic emitter, its generation mechanisms are solely determined by the light interacting with the gold band structure [35].

Our detailed analysis of the MPPL process establishes that its nonlinear order is higher than the THG. In addition we observe that the MPPL order decreases while the excitation pulse is tuned to shorter wavelengths. Figure 4 demonstrates this finding by following the same procedure discussed for the antenna resonant at $2000 \mathrm{~nm}$ in Fig. 3. For this reason we would expect to achieve more efficient MPPL by driving the antennas at, e.g., $1200 \mathrm{~nm}$. Nevertheless, as it is identifiable in Fig. 2, the opposite effect occurs and we observe that for increasing excitation wavelength the MPPL emission exceeds the THG. This counterintuitive result hints to the fact that, rather than multiphoton absorption, THG is strongly affected by the photon energy due to a pronounced dispersion of the $\chi^{(3)}$ nonlinearity in gold $[21,27]$. In order to evaluate this aspect, we compare the emission spectra acquired from a large number of individual antennas, each of them illuminated with resonant radiation. It is crucial to underline that the experimental conditions were kept as similar as possible despite the fact that the driving pulses were tuned over a broad frequency range. In detail, excitation power and duration of the pulses with well-behaved spectral phase were characterized before each set of measurements to ensure that the results are directly comparable. A selection of the tunability of the excitation spec- 

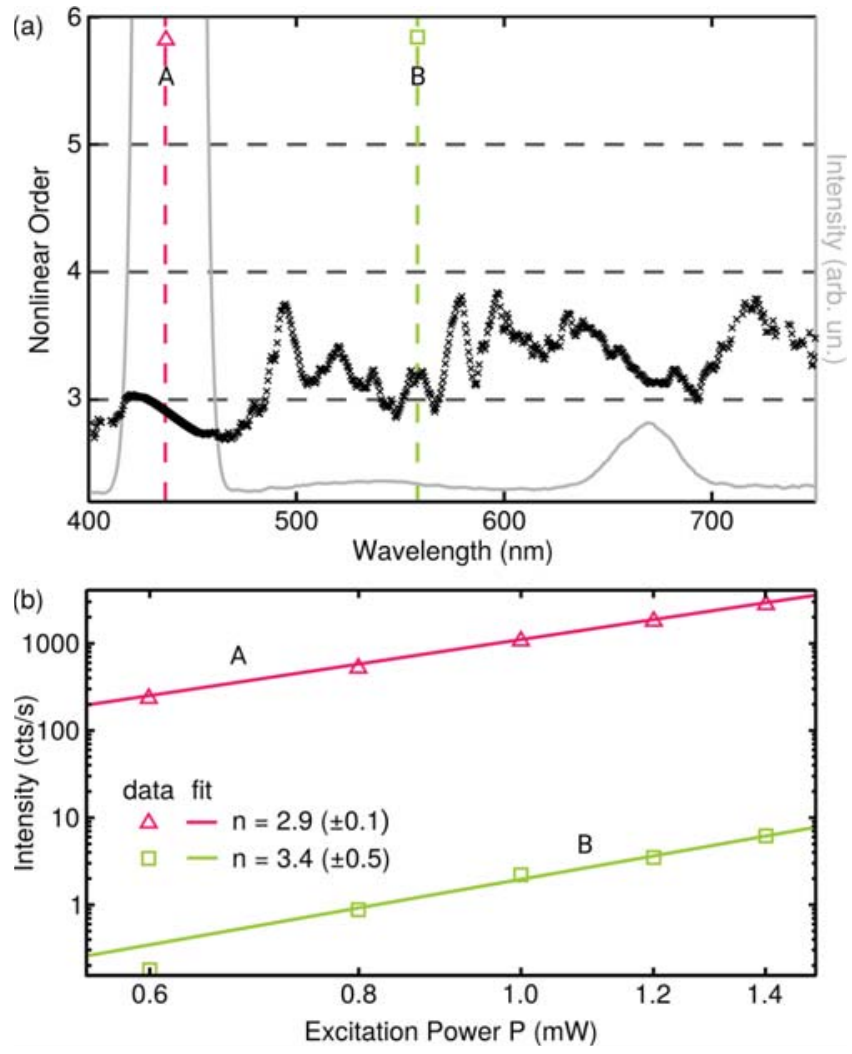

FIG. 4. Spectrally resolved power dependence of the nonlinear emission obtained from a gold nanorod of $275 \mathrm{~nm}$ length under excitation at a central wavelength of $1310 \mathrm{~nm}$. (a) The nonlinear order $n$ of each corresponding emission wavelength (black crosses) together with the nonlinear emission spectrum acquired at the maximum excitation power (gray). The vertical lines A and B mark positions for which the power dependence fits are shown in (b): the experimental data points of emission intensity are depicted versus excitation power (symbols). The solid lines in this double-logarithmic graph show the least-square exponential fits determining the nonlinear order $n$ plotted in (a).

tra is shown in Fig. 5(a). The region of the fundamental laser light $(1550 \mathrm{~nm} \pm 50 \mathrm{~nm})$ is not exploited in the measurements since the nonlinear frequency conversion that grants tunability to the laser system causes spectral modulations at the driving wavelength. Structured spectra cause long pulse pedestals in the time domain and temporal distortions influence the yield of photoluminescence since this process is affected by the thermalization dynamics of electrons [18].

Figure 5 shows the spectra [panel (a)] of the pulses used to excite individual antennas resonant at different frequencies and the resulting contribution of THG [panel (b)] and MPPL [panel (c)] to the nonlinear emission. The analysis is obtained by separating the MPPL signal from the coherent THG [as explained in Fig. 2(b)]. This procedure is repeated for each investigated specimen and the total emission is extracted by spectrally integrating the two independent components. The integration boundaries are set to 365 and $850 \mathrm{~nm}$. It is worth noticing that the yield of the two nonlinear processes shows opposite trends.

In particular, it is striking that the MPPL output grows for longer excitation wavelengths while the THG decreases.

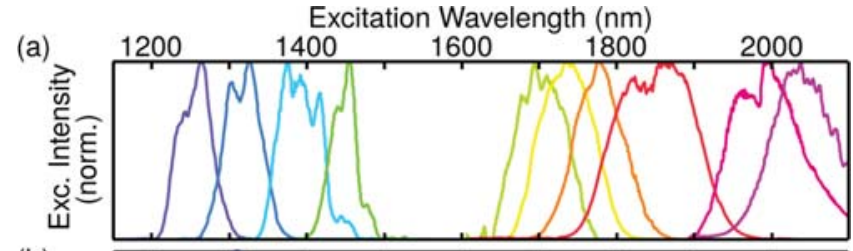

(b)

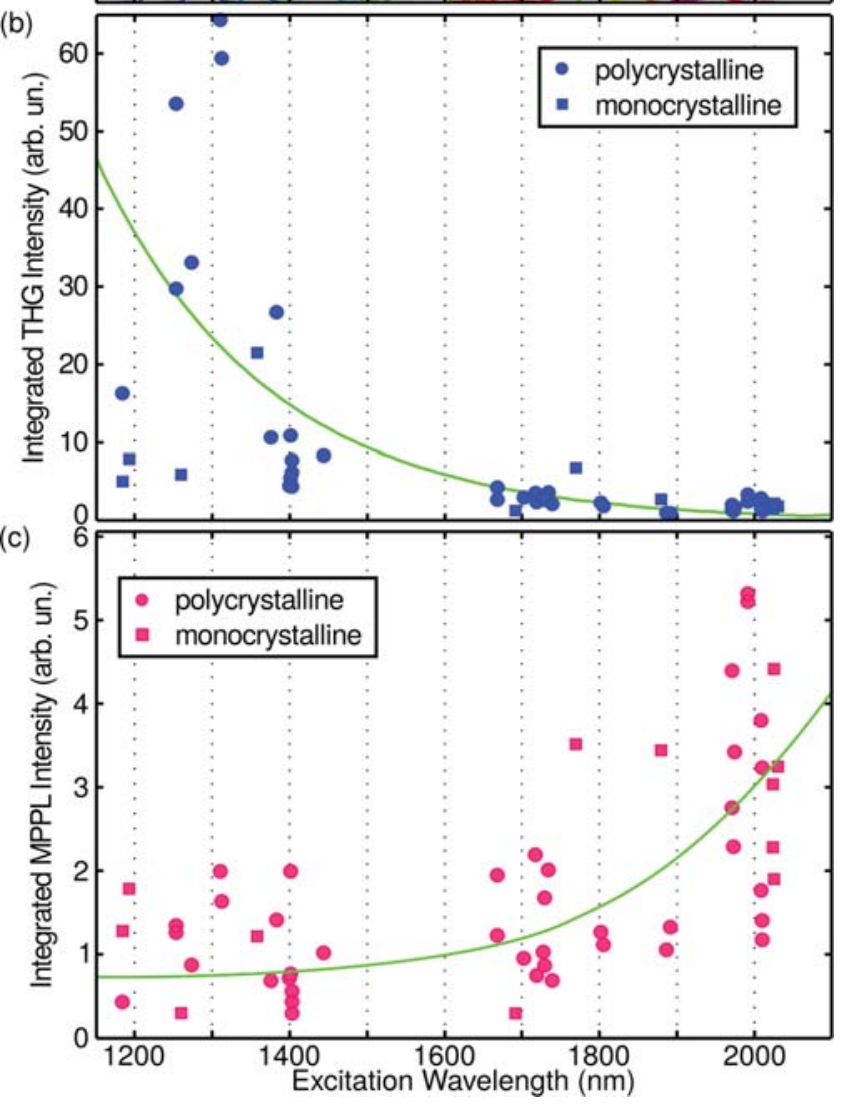

FIG. 5. (a) Selected excitation spectra that were used to obtain THG and MPPL from gold nanorods. The integrated nonlinear emission is shown as a function of the center wavelength of the driving pulse for THG (b) and MPPL (c), respectively. Each data point marks the result from a single individual antenna. Both monoand polycrystalline specimens are considered in these measurements with no significant differentiations. The green lines serve as a guide to the eye.

Even more interestingly, the THG shows strong frequency dependence and its yield is 50 fold higher when the driving frequency is set to $1200 \mathrm{~nm}$, compared to excitation at $2000 \mathrm{~nm}$. These observations are particularly remarkable if we consider that antennas resonant at shorter wavelengths have a length that is almost half of the one that is optimized for resonance at $2000 \mathrm{~nm}$. So, while the MPPL roughly scales linearly with the volume of the plasmonic emitter, the THG is following a strong dispersion in its efficiency.

Still, the experimental results display a large variability in the nonlinear emission even for antennas that are fabricated to be resonant at the same frequency since small nanoscopic defects affect the field enhancement factor, leading to strong differences in the efficiencies of nonlinear frequency conversion phenomena [20,28,29]. Figure 6 plots the ratio of THG with respect to the total nonlinear emission for 


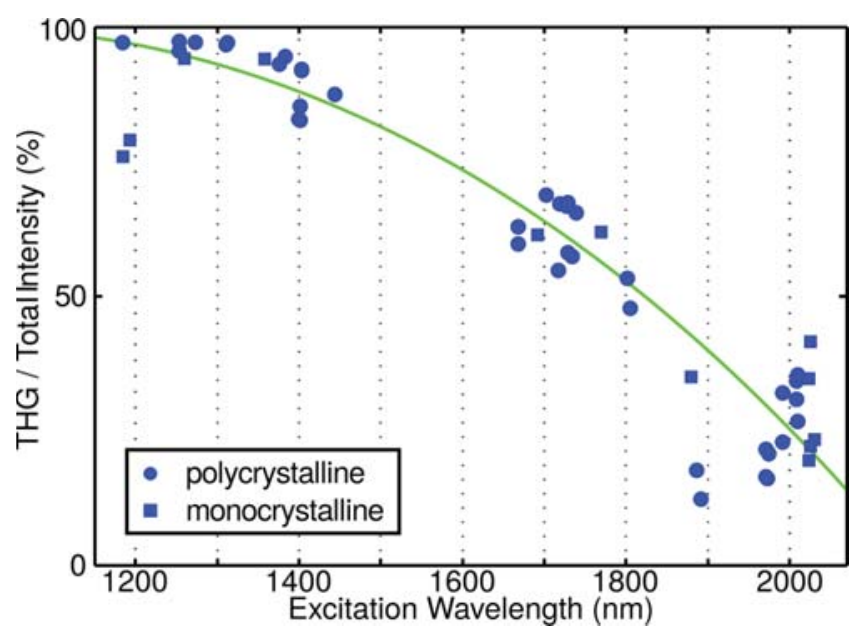

FIG. 6. Ratio between THG and total nonlinear emission. Each data point is obtained from a single gold nanorod. The green line serves as a guide to the eye.

each nanoantenna under study. This representation of the experimental data is independent of the effective value of the field enhancement and clearly demonstrates the increase in the coherent emission at the expense of MPPL for shortwavelength excitation.

Our interpretation of the frequency dependence of the THG efficiency relies on the fundamental dispersion of the $\chi^{(3)}$ nonlinearity. As already discussed, gold allows direct dipole absorption between $d$-electrons and unoccupied states above the Fermi level in the conduction band. The presence of these interband transitions shapes the polarizability of gold, since they occur even below the plasma frequency, thus affecting also its nonlinear behavior. For this reason, once the driving photon energy is tuned to be approximately one third of the $2.4 \mathrm{eV}$ energy of the interband transition [39,40], the third-harmonic emission becomes resonantly enhanced [27]. Remarkably, the efficiency of the THG nonlinearity does not peak at $1550 \mathrm{~nm}(0.8 \mathrm{eV})$ but continues to grow for shorter fundamental wavelengths. In fact, the energy of $2.4 \mathrm{eV}$ marks only the onset of a wide band of optical transitions, for the excitation of $d$ electrons to free states above the Fermi level, that concur to the resonant enhancement.

By exploiting the MPPL order to normalize the uncertainty on the field enhancement for the rods, we can estimate the relative nonlinear coefficient for three antennas, resonant at the wavelengths 1300, 1700, and $2000 \mathrm{~nm}$, respectively. The average nonlinear order $n$ of the photoluminescence is calculated by weighting the wavelength dependent quantity with the emission spectrum. The spectrally integrated intensities for the THG and PL ( $I_{\mathrm{THG}}$ and $\left.I_{\mathrm{PL}}\right)$ are considered to be proportional to: $I_{\mathrm{THG}} \propto\left|d^{(3)} E_{\text {exc }}^{3}\right|^{2}$ and $I_{\mathrm{PL}} \propto\left|E_{\text {exc }}^{n}\right|^{2}$, with $d^{(3)}$ being the nonlinear coefficient for $\chi^{(3)}$ processes defined in
TABLE I. Estimation of the relative nonlinear coefficient for the THG acquired at three different excitation wavelengths.

\begin{tabular}{lccc}
\hline \hline Excitation & $\begin{array}{c}1300 \mathrm{~nm} \\
(0.9 \mathrm{eV})\end{array}$ & $\begin{array}{c}1700 \mathrm{~nm} \\
(0.7 \mathrm{eV})\end{array}$ & $\begin{array}{c}2000 \mathrm{~nm} \\
(0.6 \mathrm{eV})\end{array}$ \\
\hline$n$ (averaged) & 3.3 & 3.9 & 3.8 \\
$d^{(3)}$ (arb. un.) & 9.8 & 4.5 & 1.7 \\
$d^{(3)} / d_{2000 \mathrm{~nm}}^{(3)}$ & 5.8 & 2.6 & 1.0 \\
\hline \hline
\end{tabular}

arbitrary units and $E_{\text {exc }}$ the electric field of the excitation pulse. We then can estimate that $d^{(3)} \propto \sqrt{I_{\mathrm{THG}} / I_{\mathrm{PL}}^{3 / n}}$. While this approach is not suitable for the exact determination of the nonlinear susceptibility, it is particularly useful to assess its dispersion as a function of the driving wavelength. In fact, we can calculate the dimensionless ratio $d_{1700 \mathrm{~nm}}^{(3)} / d_{2000 \mathrm{~nm}}^{(3)}$, which is approximately 2.6 , and $d_{1300 \mathrm{~nm}}^{(3)} / d_{2000 \mathrm{~nm}}^{(3)}$, yielding 5.8. These results are summarized in Table I and highlight the enhancement of the THG process occurring at short fundamental wavelengths.

A final consideration has to be made about polycrystalline and monocrystalline antennas. In both cases the relative differences between the MPPL and THG show the same trends as discussed above. It should be noted, however, that the most intense nonlinear emission was typically achieved with polycrystalline specimens. This observation might be simply related to the stronger field enhancement in a nanostructure where the surface presents a more pronounced corrugation, intrinsically related to the polycrystallinity [20].

In conclusion, we have experimentally proven that the coherent nonlinear emission in gold nanoantennas displays a strong dispersion that is compatible with the enhancement occurring when the third-harmonic photons become resonant with dipole-allowed transitions. Additionally, we observe that the efficiency of MPPL follows the opposite trend. These results explain the large variability in the past determination of the optical nonlinearity of gold. Furthermore, in this work we also prove that the direct comparison of coherent and incoherent nonlinear emission can be exploited to overcome the intrinsic difficulty in assessing the field enhancement of nanostructures. The capability to extract fundamental insight in the nonlinear behavior of metallic nanosystems is crucial when exploiting the full potential of plasmonic devices. In fact, while these advanced tools are plagued by large losses in linear regimes, they might display their versatility in nonlinear applications as localized light sources at subdiffraction scales.

This work was funded by the DFG via collaborative research center SFS767. D.B. acknowledges support of Zukunftskolleg, of Baden-Württemberg Stiftung through the Eliteprogramm and of the EU through the Marie Curie CIG project "UltraQuEsT" No. 334463.
[1] E. Ozbay, Science 311, 189 (2006).

[2] T. Hanke, J. Cesar, V. Knittel, A. Trügler, U. Hohenester, A. Leitenstorfer, and R. Bratschitsch, Nano Lett. 12, 992 (2012).
[3] P. Biagioni, J.-S. Huang, and B. Hecht, Rep. Prog. Phys. 75, 024402 (2012).

[4] P. Bharadwaj, B. Deutsch, and L. Novotny, Adv. Opt. Photon. 1, 438 (2009). 
[5] A. Kinkhabwala, Z. Yu, S. Fan, Y. Avlasevich, K. Müllen, and M. E. Moerner, Nat. Photon. 3, 654 (2009).

[6] P. J. Schuck, D. P. Fromm, A. Sundaramurthy, G. S. Kino, and W. E. Moerner, Phys. Rev. Lett. 94, 017402 (2005).

[7] K. D. Ko, A. Kumar, K. H. Fung, R. Ambekar, G. L. Liu, N. X. Fang, and K. C. Toussaint, Jr., Nano Lett. 11, 61 (2011).

[8] A. Bouhelier, M. Beversluis, A. Hartschuh, and L. Novotny, Phys. Rev. Lett. 90, 013903 (2003).

[9] K. B. Crozier, A. Sundaramurthy, G. S. Kino, and C. F. Quate, J. Appl. Phys. 94, 4632 (2003).

[10] A. Sundaramurthy, K. B. Crozier, G. S. Kino, D. P. Fromm, P. J. Schuck, and W. E. Moerner, Phys. Rev. B 72, 165409 (2005).

[11] T. Hanke, G. Krauss, D. Träutlein, B. Wild, R. Bratschitsch, and A. Leitenstorfer, Phys. Rev. Lett. 103, 257404 (2009).

[12] N. Pfullmann, C. Waltermann, M. Kovačev, V. Knittel, R. Bratschitsch, D. Akemeier, A. Hütten, A. Leitenstorfer, and U. Morgner, Appl. Phys. B 113, 75 (2013).

[13] C. Ropers, D. R. Solli, C. P. Schulz, C. Lienau, and T. Elsaesser, Phys. Rev. Lett. 98, 043907 (2007).

[14] M. Danckwerts and L. Novotny, Phys. Rev. Lett. 98, 026104 (2007).

[15] V. Kravtsov, R. Ulbricht, J. M. Atkin, and M. B. Raschke, Nat. Nanotechol. 11, 459 (2016).

[16] P. Mühlschlegel, H.-J. Eisler, O. J. Martin, B. Hecht, and D. W. Pohl, Science 308, 1607 (2005).

[17] P. Biagioni, M. Celebrano, M. Savoini, G. Grancini, D. Brida, S. Mátéfi-Tempfli, M. Mátéfi-Tempfli, L. Duò, B. Hecht, G. Cerullo, and M. Finazzi, Phys. Rev. B 80, 045411 (2009).

[18] P. Biagioni, D. Brida, J.-S. Huang, J. Kern, L. Duò, B. Hecht, M. Finazzi, and G. Cerullo, Nano Lett. 12, 2941 (2012).

[19] X.-F. Jiang, Y. Pan, C. Jiang, T. Zhao, P. Yuan, T. Venkatesan, and Q.-H. Xu, J. Phys. Chem. Lett. 4, 1634 (2013).

[20] G. T. Boyd, Z. H. Yu, and Y. R. Shen, Phys. Rev. B 33, 7923 (1986).

[21] R. W. Boyd, Z. Shi, and I. de Leon, Opt. Commun. 326, 74 (2014).

[22] W. K. Burns and N. Bloembergen, Phys. Rev. B 4, 3437 (1971).
[23] D. D. Smith, Y. K. Yoon, R. W. Boyd, J. K. Campbell, L. A. Baker, R. M. Crooks, and M. George, J. Appl. Phys. 86, 6200 (1999).

[24] N. Rotenberg, A. D. Bristow, M. Pfeiffer, M. Betz, and H. M. van Driel, Phys. Rev. B 75, 155426 (2007).

[25] J. Renger, R. Quidant, N. van Hulst, and L. Novotny, Phys. Rev. Lett. 104, 046803 (2010).

[26] F. Hache, D. Ricard, C. Flytzanis, and U. Kreibig. Appl. Phys. A 47, 347 (1988).

[27] M. Conforti and G. Della Valle, Phys. Rev. B 85, 245423 (2012).

[28] A. Bouhelier, R. Bachelot, G. Lerondel, S. Kostcheev, P. Royer, and G. P. Wiederrecht, Phys. Rev. Lett. 95, 267405 (2005).

[29] O. Demichel, M. Petit, S. Viarbitskaya, R. Méjard, F. de Fornel, E. Hertz, F. Billard, A. Bouhelier, and B. Cluzel, ACS Photon. 3, 791 (2016).

[30] M. Lippitz, M. A. van Dijk, and M. Orrit, Nano Lett. 5, 799 (2005).

[31] M. Eichelbaum, B. E. Schmidt, H. Ibrahim, and K. Rademann, Nanotechnology 18, 355702 (2007).

[32] R. A. Farrer, F. L. Butterfield, V. W. Chen, and J. T. Fourkas, Nano Lett. 5, 1139 (2005).

[33] M. R. Beversluis, A. Bouhelier, and L. Novotny, Phys. Rev. B 68, 115433 (2003).

[34] R. Méjard, A. Verdy, M. Petit, A. Bouhelier, B. Cluzel, and O. Demichel, ACS Photon. 3, 1482 (2016).

[35] V. Knittel, M. P. Fischer, T. de Roo, S. Mecking, A. Leitenstorfer, and D. Brida, ACS Nano 9, 894 (2015).

[36] A. Sell, G. Krauss, R. Scheu, R. Huber, and A. Leitenstorfer, Opt. Express 17, 1070 (2009).

[37] D. Brida, G. Krauss, A. Sell, and A. Leitenstorfer, Laser \& Photon. Rev. 8, 409 (2014).

[38] B. Radha and G. U. Kulkarni, Curr. Sci. 102, 70 (2012).

[39] N. E. Christensen and B. O. Seraphin, Phys. Rev. B 4, 3321 (1971).

[40] M. Guerrisi, R. Rosei, and P. Winsemius, Phys. Rev. B 12, 557 (1975). 